\title{
Biliary Fistula, CTCAE
}

National Cancer Institute

\section{Source}

National Cancer Institute. Biliary Fistula, CT CAE. NCI Thesaurus. Code C143316.

A disorder characterized by an abnormal communication between the bile ducts and another organ or anatomic site. 